\title{
5. SOLAR FLARES AS A PINCH-EFFECT
}

\author{
A. B. SEVERNY
}

Flares are extremely non-stationary phenomena. We will summarize the properties of some flares examined at high dispersion and resolving power with the solar tower telescope in the Crimea.

(I) The spectral emission of all flares is concentrated in very small $\left(0^{\prime \prime} 5^{-2}\right.$ ") shortlived $\left(4^{\mathrm{m}}-20^{\mathrm{m}}\right)$ grains. These may be likened to moustaches, the phenomenon described by the author at the last General Assembly of the I.A.U. at Dublin [r].

(2) All flares may be divided into two classes: (a) usual 'chromospheric' flares without moustaches (generally of importance $<2$ ) and $(b)$ those possessing broad Io-I5 $\AA$ emission wings in flash-phase (importance $>2$ ). We have found that under good seeing conditions these wings can be resolved into separate moustaches [2].

(3) In both $A$ and $B$ types we found that Balmer emission lines are all broadened by Stark-effect. From investigation of line profiles we found the following data for the physical state of plasma at the periphery of a flare.

$$
N_{e} \simeq N_{H}=\mathrm{IO}^{13}, \quad T_{\mathrm{k} \ln }=\mathrm{IO}^{4}-3 \cdot \mathrm{IO}^{4}{ }^{\circ} \mathrm{K} .
$$

The calculations of different processes of excitation, de-excitation, ionization and recombination for different quantum levels of hydrogen in such a plasma have led us to the conclusion that the electron gas in flares cools very rapidly (within a fraction of a second) owing to cascade ionizations and excitations by electronic impact. There must exist a source maintaining the energy of electrons at the rate $500 \mathrm{ergs} / \mathrm{cm} / \mathrm{sec}$ to prevent such rapid cooling [3].

(4) The Hydrogen and $\mathrm{Ca}$ II line profiles of more than thirty isolated moustaches as well as the moustaches in big flares are in perfect agreement with Doppler-broadening produced in optically thin layers by macroscopic motions ('turbulence') with velocities $50<v<250 \mathrm{~km} / \mathrm{sec}$. The most convincing evidence for this was from the flare above the limb of 1957 September 9 [4].

(5) We also would like to report: (a) the strengthening of the $\mathrm{D} \alpha$-line in moustaches and several flares of A type, which we observed in twenty-five cases, indicating the possibility of formation of neutrons [s]; $(b)$ the well-known indications of streams of corpuscles with velocities of $50-100$ thousand $\mathrm{km} / \mathrm{sec}$ appearing in the interpretation of radio-bursts; $(c)$ the generation of cosmic rays with energies $\simeq \mathrm{IO}^{9} \mathrm{eV}$ in several cases; (d) the D-layer response on flares and I.G.Y.-rocket measurements showing that X-rays in the main are generated in flares but not $L \alpha[6]$.

(6) In the last two years we have paid a great deal of attention to solar magnetograph measurements of magnetic fields in active regions [7]. These measurements show the remarkable fine structure, sometimes I" -2 ", of these fields. They are not of dipole character, but represent something like a bunch of moving and entangled snakes-tubes of lines of force. Fields of considerable strength (up to 500 gauss) extend into the upper layers of the chromosphere [8]. But the most interesting relates to flares. We found that flares at their very beginning appear at neutral points of sunspot magnetic field when the gradient of field strength is sufficiently high in the vicinity of such points. We found that the appearance of a flare leads to the destruction and rearrangement of the surrounding magnetic field $[4,9]$.

(7) It can be shown that a plasma must be unstable when the concentration of lines of forces frozen into plasma around a neutral point is sufficiently high. This is because the magnetic force contracting the plasma increases more rapidly than the pressure gradient, and thus the plasma can contract without limit. There is a pinch-effect in the homogeneous current layer and the similar phenomena can be observed in laboratory experiments. However, it can easily be shown that at the stage $x=0.1$ of the contraction ( $x$ being the ratio of dimensions $a / a_{0}$, where $a_{0}$ is the original characteristic dimension) shock-fronts converging to the neutral plane are developing. At the stage $x=0 \cdot 01$ the reaction of the 


\title{
JOINT DISCUSSION
}

shock wave beyond the front causes the contraction to stop and makes it expand-the phenomenon which is often observed after the onset of flares. The calculation shows that the collision of two shock-fronts at neutral plane can heat the plasma impulsively up to $10^{7} \mathrm{~K}$ in a small region $\left(\simeq 10^{5} \mathrm{~cm}\right)$, sets up powerful macroscopic motions with velocities more than $100 \mathrm{~km} / \mathrm{sec}$, and leads to the destruction (diffusion) of the initial magnetic field, which correspond to the observed phenomena of moustaches and of the destruction of magnetic fields by flares.

Two shock fronts approaching each other with velocity $\simeq 6.10^{7}$ can be considered as two magnetic mirrors, because $w_{e} \tau_{e} \gg \mathrm{I}$ in the fronts as well as in space between them. The gain of energy by electrons trapped in this space increases like $1 / a^{2}$ ( $a$ being the distance between the fronts) and the leading term in the energy losses will increase as $1 / a$ (ionization losses). X-rays and $\gamma$-radiation, as the result of Bremsstrahlung of accelerated particles and impulsive heating, should accompany the streams of electrons. This is in agreement with rocket measurements. It is of interest to note that it is possible to bring into quantitative agreement the data about the continuous emission of flares in the visible part of the spectrum with these rocket measurements when we assume that this visible continuous emission is the 'long wave-length tail' of Bremsstrahlung of particles with initial energies Io-IOO $m_{0} c^{2}$. This shows that flares may be also considered as a source of $\gamma$-radiation [ro].

\section{REFERENCES}

[1] Severny, A. B. Trans. I.A.U. 9, 154, 1955; Publ. Crim. astrophys. Obs. 16, 194, 1956.

[2] Severny, A. B. Astr. J., Moscow, 34, 684, 1957.

[3] Severny, A. B. Publ. Crim. astrophys. Obs. 19, 72, 1958.

[4] Severny, A. B. Astr. J., Moscow, 35, 335, 1958.

[5] Severny, A. B. Ibid. 34, 328, 1957.

[6] Friedman, H. et al. I.G.Y. Project, 1957.

[7] Nikulin, N. S., Severny, A. B. and Stepanov, V. E. Publ. Crim. astrophys. Obs. 19, 3, I958.

[8] Bumba, V. and Severny, A. B. Observatory, 78 (no. 902), 33, 1958.

[9] Severny, A. B. Publ. Crim. astrophys. Obs. 20, 22, 1958.

[10] Severny, A. B. C.R. Acad. Sci. U.R.S.S. r21, 819, 1958.

\section{FLARE-ASSOCIATED PHENOMENA IN THE SOLAR CORONA}

\author{
WALTER ORR ROBERTS
}

\section{INTRODUCTION}

The solar corona is markedly affected by the occurrence of flares. From a study of flarecorona interactions we may, thus, gain improved insight into the physical conditions responsible for flares and their terrestrial consequences. Only the beginnings of such researches have yet been made.

Observational studies of the associated coronal effects of flares, though less abundant and less detailed than we would like, do however indicate several salient facts:

(a) Pronounced apparent motions often occur in the emission-line corona at the times and locations of flares.

(b) When suitable observations of limb flares are available they seem to imply that tightly-curved prominences of looped character (type $\mathrm{AS}_{1}$ in the Menzel and Evans classification [r]) are intimately connected with the flares, if indeed they are not the flares themselves. This tightly-looped character is also evident in the line-emission corona, though simultaneous prominence and coronal photographs show that the motions differ markedly. 